\title{
Poly(vinyl alcohol)-Salicylic Acid Conjugate: Synthesis and Characterization
}

\author{
Roman Jantas*, Zbignie w Draczyński, Lucyna He rczyńs ka, Dawid Stawski \\ Department of Phy sical Chemistry of Polymers, Technical University of Łódź, 90-924, Łódź, Poland
}

\begin{abstract}
The polymeric system containing hydrolysable ester bonds linked to salicylic acid was synthesized for controlled drug release. Poly(vinyl alcohol) (PVA) functionalized with chloroacetate groups was obtained by reaction of PVA with chloroacetyl chloride using the $N, N$-dimethylacetamide/5\% lithium chloride system as solvent and pyridine as catalyst. The degree of substitution was calculated from the chloride content and ranged from 37.8 to 98.9 mol\%. The coupling of salicylic acid to PVA functionalized with chloroacetate groups was carried out by the reaction with between PVA and the sodium salt of salicylic acid. The structures of chloroacetylated PVA and PVA-salicylic acid conjugates were determined by means FTIR, ${ }^{1} \mathrm{H}-\mathrm{NMR}$ and ${ }^{13} \mathrm{C}$-NMR spectra. The hydrolysis in the heterogeneous system of PVA-salicylic acid conjugates were performed in buffer solutions ( $\mathrm{pH} 7.6$ and 8.5 ) at $37^{\circ} \mathrm{C}$. Detection of hydrolysis by UV spectroscopy showed that released the drug, can by achieved by the hydrolys is of the ester bond between the drug and the poly meric carrier. The release profiles indicated that the release of the drug (sodium salicylate) from tablets was dependent on hydrophilic character of conjugate and the $\mathrm{pH}$ of the buffer solution.
\end{abstract}

Keywords Poly(Vinyl Alcohol), Chloroacetylation, Sodium Salicylate, Poly mer-Drug Conjugate, Controlled Release

\section{Introduction}

In the last decades much attention has been directed to speciality polymers for biomedical application. One particular approach towards the improved use of drugs is the design of polymer-drug conjugates or polymeric prodrugs[1-9]. The chemical attachment of low molecular weight drugs to synthetic or natural polymers has been frequently investigated as a means of improving the efficacy of drug control release devices through a constant but prolonged release of drugs with minimum side effects. The drugs may be linked to the polymeric carriers using a number of chemical reactions with participation of functional groups such as hydroxyl or carboxyl which are either originally present in the polymer chain or alternatively formed by functionalization. Another possibility is the use of functionalized monomers in synthesis of a reactive polymeric precursor. In most cases, drugs bound directly to the polymer chain exhibit either a reduced or zero biological activity. For this reason, drugs should be separated from the poly meric backbone by means of a spacer. Once the drug conjugate reaches the target compartment, the drug can then be split off more readily in its active form. To facilitate the release of the drug it must

* Corresponding author:

rojan@p.lodz.pl (Roman Jantas)

Published online at http://journal.sapub.org/ajps

Copyright (C) 2012 Scientific \& Academic Publishing. All Rights Reserved be attached to the macro molecu lar chain by covalent bonds of limited stability in a bio logical environ ment.

The advantages of poly(ethylene oxide), poly(vinyl pyrrolidone), poly(2-hydroxypropyl methacrylamide), copolymers of 2-hydroxyethyl methacrylate or polysaccharides as a macromolecular carrier for drug immobilization are well acknowledged, as is apparent from the literature data[10-19]. In most cases the polymers has been previously transformed into a suitable reactive derivative, in order to achieve the attachment of drug molecules and to introduce a spacer between the carrier and the bioactive compounds. Selection of the spacer arm is critical as it opens the possibility of controlling the site and the rate of release of the drug from the conjugates either by hydrolytic or enzy mat ic cleavage of the lin king bond.

Some natural or synthetic polymers possess multiple primary and secondary hydroxyl groups and there can be easily conjugated with drug molecules with reactive groups either by direct conjugation or by incorporation of a spacer arm. In this paper, PVA with reactive primary hydroxyl groups may be used as a polymeric carrier for coupling pharmaceutical compounds.

Salicylates are used in medicine as analgesic and antipy retic agents. They also act as non-steroidal anti-in fla mmantory drugs (NSAIDs) and induces apoptosis in cancer cells [20-23]. Recent years have witnessed many studies on the synthesis and hydrolysis of polymer-drug conjugates of NSAIDs such as: naproxen[12,24] indomethacin[10,25,26] ibuprofen[27,28] ketoprofen[12,29] and fenoprofen[30-32]. 
The aim of the present paper was to synthesize and characterize PVA-salicylic acid conjugates in a two-stage procedure. During the first stage, PVA was chloroacetylated with chloroacetyl chloride, while in the second stage, chloroacetate groups were reacted with sodium salicylate. A study of the hydrolysis of the resulting conjugates in the heterogeneous system was also conducted in aqueous buffer solutions (pH 7.6 and 8.5 ) and the quantity of the released drug was detected by UV spectroscopy. The influence of neighbouring groups on the release of the drug from the conjugates was also studied.

\section{Experimental}

\subsection{Materials}

Poly(vinyl alcohol) (PVA) was commercial product (Aldrich). The average molecular weight of PVA was $\mathrm{M}_{\mathrm{W}}=31.600-50.000 \mathrm{~g} / \mathrm{mol}$, 98-99\% hydrolyzed. N,Ndimethylacetamide (DMAc) (Aldrich), dimethylsulfoxide (DMSO) (Aldrich) was purified by distillation and then stored over $4 \AA$ moleculare sieves. Lithium chloride $(\mathrm{LiCl})$ (Aldrich) was dried under reduced pressure in the presence of phosphorus pentoxide. Chloroacetyl chloride (Aldrich) was purified before use by distillation under reduced pressure. Pyridine (POCh) was refluxed over $\mathrm{CaH}_{2}$ under a nitrogen atmosphere and then distilled. Salicylic acid (SA) (Fluka, Buchs, Switzerland) was used without further purification. Sodium salicylate (SSA) was obtained by dissolving $8.05 \mathrm{~g}(0.05 \mathrm{~mol})$ of the acid in $50 \mathrm{ml}$ of chloroform, then neutralized with $2.0 \mathrm{~g}(0.05 \mathrm{~mol})$ of $\mathrm{NaOH}$ dissolved in $50 \mathrm{ml}$ of ethyl alcohol. The product precipitated by pouring reaction mixtu re into $600 \mathrm{ml}$ of dry acetone. After filtration, the salt was dried under reduced pressure at $50^{\circ} \mathrm{C}$ to constant weight.

\subsection{Procedures}

\subsubsection{Esterification of PVA with Chloroacetyl Chloride}

The typical procedure, of the esterification was as follow: $2.2 \mathrm{~g}$ (50.0 mmol, OH groups) of PVA was dissolved in 30 ml DMAc/ 5\% LiCl solvent system. The solution was then charged into a three-necked flask equipped with a nitrogen inlet and outlet, dropping funnel, magnetic stirrer and thermo meter. Pyridine $4.68 \mathrm{ml}(60.0 \mathrm{mmol})$ was added to the flask as an acid acceptor. DMAc solution $(10 \mathrm{ml})$ containing chloroacetyl chloride $5.76 \mathrm{ml}(60.0 \mathrm{mmol})$ was then added dropwise at about $0^{\circ} \mathrm{C}$ with stirring. The reaction mixture was heated at $25^{\circ} \mathrm{C}$ for $8 \mathrm{~h}$ and after the solution was poured into a large amount of cold $2 \mathrm{M}$ HCl to precip itate the product. The precipitated product was filtered and washed several times with cold distilled water. It was purified by reprecipitation using THF as solvent and cold distilled water as precipitant, then dried under reduced pressure at $50^{\circ} \mathrm{C}$ to constant weight. The yield was $79 \%$.

2.2.2. Reaction of Chloroacetylated PVA with Sodium

\section{Salicylate}

The typical procedure, of the reaction was as follow: The chloroacetylated PVA $2.1 \mathrm{~g}$ (19.5 mmol $\mathrm{ClCH}_{2} \mathrm{CO}$ - groups) was dissolved in $40 \mathrm{ml} \mathrm{DMSO}$ at room temperature and then $3.68 \mathrm{~g}$ ( $23 \mathrm{mmol}$ ) sodium salicy late was added while stirring. The reaction was performed at $30^{\circ} \mathrm{C}$ and under stirring for about $5 \mathrm{~h}$. The obtained product was isolated by precipitation using distilled water as precipitant and then ethanol washed to remove unreacted sodium salicylate. All samples were purified by reprecipitation, using DMSO as solvent and ethanol as precipitant and then dried under reduce pressure at $60{ }^{\circ} \mathrm{C}$ to constant weight. The yield was $69 \%$.

\subsubsection{Study of The Heterogeneous Hydrolysis of PVA-Salicylic Acid Conjugate}

Samples of the PVA-salicylic acid conjugate, about $0.1 \mathrm{~g}$ (containing from 37.8 to 98.9 mol-\% salicylate groups) in the form of powder were pressed in steel cylindrical cell with a diameter of $12 \mathrm{~mm}$ in a hydraulic press under a pressure of about $12 \mathrm{MPa}$ to make disks. The resulting tablet was placed in conical flasks with $100 \mathrm{ml}$ of an aqueous buffer solution (pH 7.6 and 8.5). The flaskes were put into water bath heated to $37^{\circ} \mathrm{C} .2 \mathrm{ml}$ samples were taken at appropriate intervals from the liquid above of the tablets and equal volume of same dissolution medium was added to maintain a constant volume. The solution contained the released drug, which was quantitatively determined by UV spectroscopy at the absorption wavelength of sodium salicylate $(\lambda 295 \mathrm{~nm})$ using calibration curves obtained previously under the same conditions. Tests were performed for different degrees of substitution of the conjugates and various $\mathrm{pH}$ values of the reaction medium. We noticed that of PVA-salicylic acid conjugates remained insoluble in the reaction environment along the whole hydrolysis process investigated.

\section{Measurements}

\subsection{Spectroscopic Measurements}

Infrared spectra were recorded using Perkin-Elmer 2000 (FTIR) instrument. ${ }^{1} \mathrm{H}-\mathrm{NMR}$ and ${ }^{13} \mathrm{C}$-NMR spectra were obtained using Bruker DPX $250 \mathrm{MHz}$ spectrometer with DMSO- $\mathrm{d}_{6}$ as solvents and TMS as an internal reference. The UV-VIS spectra were obtained using Perkin Elmer UV/VIS Lambda 2 spectrometer.

\subsection{Evaluation the Degree of Esterification}

The degree of the esterification of the PVA was determined from the elemental analysis of chloride. Elemental analysis (Cl) was carried out on a Carlo Erba 1106 EA-instrument.

\section{Results and Discussion}

In order to provide a uniform distribution of chloro methyl 
groups along the polymer chain, the esterification was carried out in homogeneous system, previously dissolving PVA in DMAc/5\% $\mathrm{LiCl}$ system. PVA modified with chloroacetate group with different degress of substitution was synthesized using the method described for chloroacetylation of starch [19] according to the reaction presented by the Scheme 1 .<smiles>CCC(C)OC(=O)CCl</smiles>

Scheme 1. Reaction between PVA and chloroacetyl chloride

The effect of reaction conditions on degree of substitution is summarized in Table 1. The degree of the substitution of PVA was calculated from the content of chlorine determined by the elemental analysis. As follows from the date in Table 1 , the extent of modification increases with an increase in the ratio of chloroacetyl chloride to PVA. For example, the degrees substitution increases from 37.8 to $98.9 \mathrm{~mol} \%$ chloroacetate groups as chloroacetyl chloride/hydroxyl groups of PVA increase from 0.4 to 1.2 .

Table 1. Effect of reaction conditions on the degree of substitution for the esterification of PVA with chloroacetyl chloride at $25^{\circ} \mathrm{C}$

\begin{tabular}{|c|c|c|c|}
\hline Sample & $\begin{array}{c}\mathrm{ClCH}_{2} \mathrm{COCl} \\
/-\mathrm{OH} \\
\text { mole/mole }\end{array}$ & $\begin{array}{c}\mathrm{Cl} \\
\%\end{array}$ & $\begin{array}{c}\text { Degree of } \\
\text { substitution } \\
\text { mol \% }\end{array}$ \\
\hline 1 & 0.4 & 18.42 & 37.8 \\
\hline 2 & 0.8 & 26.05 & 73.6 \\
\hline 3 & 1.2 & 29.25 & 98.9 \\
\hline
\end{tabular}

The coupling of bioactive carboxylic acid to PVA functionalized with chloroacetate groups was carried out by using the sodium salicylate according to the following Scheme 2.

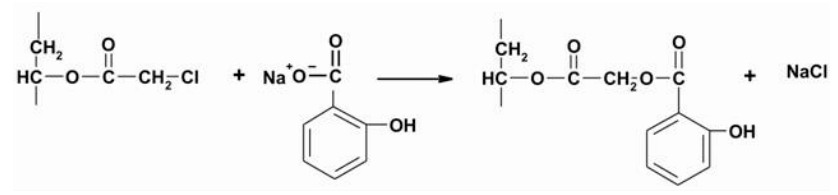

Scheme 2. Synthesis of PVA-salicylic acid conjugate

The elementary analysis of the products obtained from chloroacetylated PVA with various degrees of substitutions and sodium salicylate showed the absence of chlorine, which allowed one to assume its total replacement with the substitution degree of the conjugate being the same as that for corresponding chloroacetylated derivatives of PVA.

Figure 1a-c shows exemplary the FTIR spectra of unmodified PVA, chloroacetylated PVA (98.9 mol\% of chloroacetate groups) and PVA-salicylic acid adduct ( $98.9 \mathrm{~mol} \%$ of salicylate groups). As is seen, the spectrum of chloroacetylated PVA (Fig. 1b), unlike the spectrum of PVA (Fig. 1a), has a new absorption band at $1749 \mathrm{~cm}^{-1}$ of carbonyl group (in $-\mathrm{COO}-\mathrm{CH}_{2}-\mathrm{Cl}$ ). On the other hand, there disappears the band within the range $3934-3099 \mathrm{~cm}^{-1}$ derived from hydro xyl groups. Moreover in spectrum of the conjugate PVA-salicylic acid (Fig. 1c) absorption band appears at 1683, 1615, 1585 and $761 \mathrm{~cm}^{-1}$, which results from scissoring vibrations bands of $>\mathrm{C}=\mathrm{C}<$ and $\mathrm{C}-\mathrm{H}$ in the benzene ring.

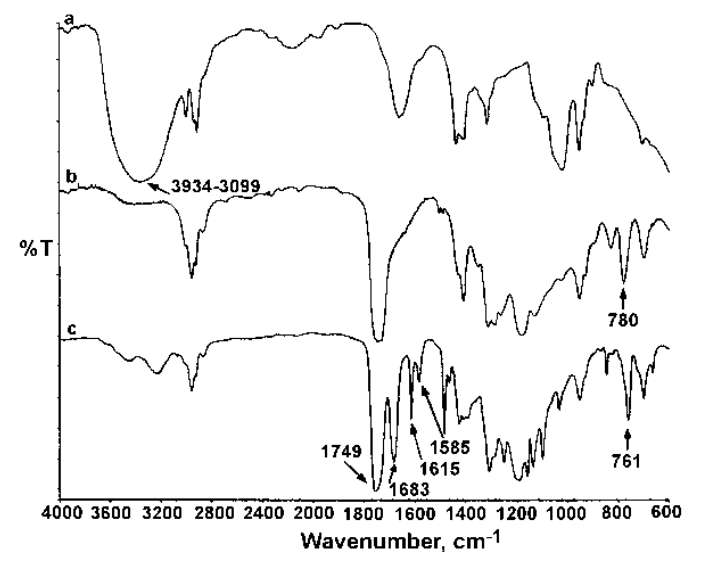

Figure 1. FTIR spectra of: a - PVA, b - chloroacet ylat ed PVA ( $98.9 \mathrm{~mol} \%$ of chloroacet ate groups), c - conjugate of PVA-salicylic acid (98.9 mol\% of salicylate groups)

The ${ }^{1} \mathrm{H}$-NMR spectrum of the same chloroacetylated PVA (Fig. 2a) shows a characteristic peak of protons of chloroacetate groups at $4.05 \mathrm{ppm}$. There is also visible peak at $1.72 \mathrm{ppm}$, which belong to protons of $-\mathrm{CH}_{2}$ - in the main chain. The spectrum of PVA-salicylic acid conjugate shows characteristic signals at $6.72-7.83 \mathrm{ppm}$ and at $10.2 \mathrm{ppm}$, which can be assigned to the protons of the benzene ring and -OH groups, respectively.

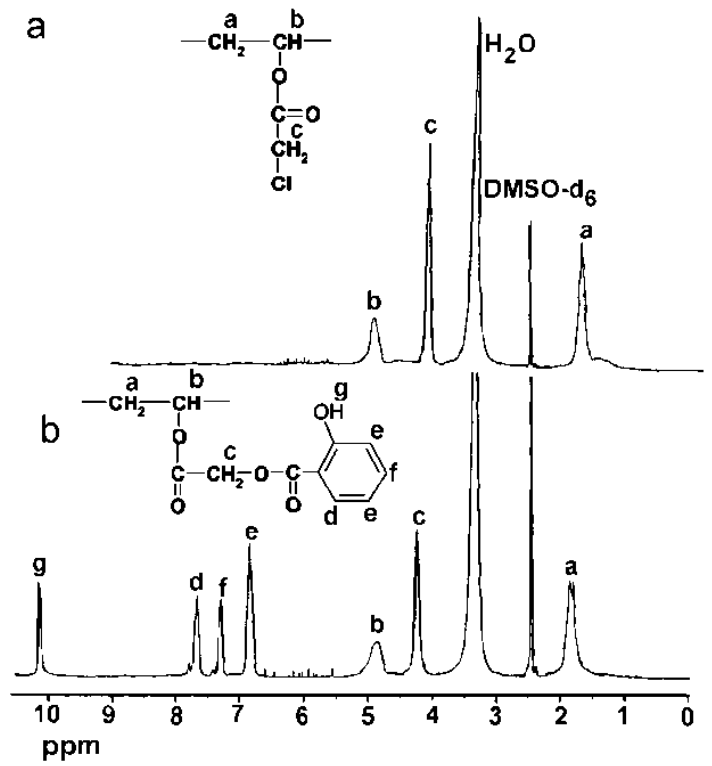

Figure 2. ${ }^{1} \mathrm{H}-\mathrm{NMR}$ spectra of: a - chloroacetylated PVA $(98.9 \mathrm{~mol} \%$ of chloroacetate groups), b - conjugate of PVA-salicylic acid (98.9 mol\% of salicylate groups)

The ${ }^{13} \mathrm{C}-\mathrm{NMR}$ spectrum of chloroacetylated PVA is characterized by chemical shifts at 39.8 and $166.1 \mathrm{ppm}$, which correspond to chloromethyl and carbonyl carbon atoms of chloroacetate groups, respectively. The spectrum of 
the PVA-salicylic acid conjugate shows additional peaks between 111.4 and $134.9 \mathrm{ppm}$, which due to the resonance of carbon atoms in the benzene ring and the signals at 158.4 ppm can be assigned to the $\mathrm{C}_{6} \mathrm{H}_{4}$ - $\mathrm{CO}$ - groups. All these spectroscopic results confirm the presence of chloroacetate and salicylate groups in the modified PVA.

From the literature reports it follows that the side chain hydrolysis of drug pendent polymers depends on the strength and chemical nature of the polymer-drug bonds, the structure of polymer and medium conditions. The hydrolysis of the bond is also dependent on its distance fro $m$ the ma in poly mer chain. The length and hydrophilicity of the spacer between the drug and polymer backbone can also influence the drug release rate[33]. The in vitro hydrolysis behavior of polymer-drug conjugate was studied in buffer solutions $\mathrm{pH}$ 7.6 and 8.5 ) at $37^{\circ} \mathrm{C}$. Two hydrolyzable ester groups were present in PVA-salicylic acid conjugates. The investigation of the hydrolyzing solution by UV spectroscopy showed that the polymer-drug conjugates released the sodium salicylate gradually under mild conditions, by the hydrolysis of the ester bond between the drug and side chain of the polymer during the reaction. The direct ester lin kage to the ma in chain of the polymer was less susceptible towards hydrolysis. This may be connected with the steric hindrance of the poly meric chain that reduces the bond mobility[34].

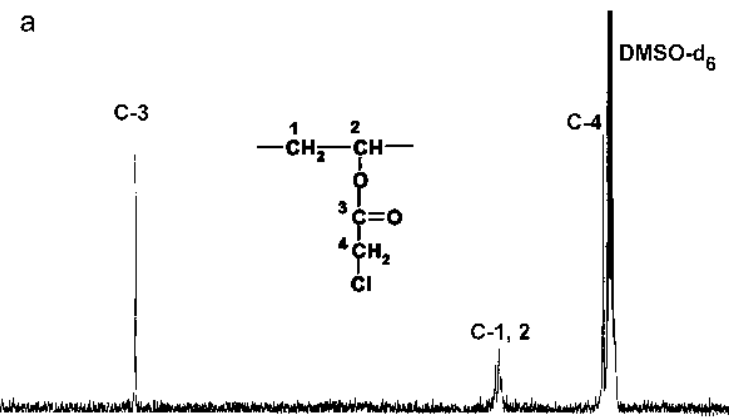

b
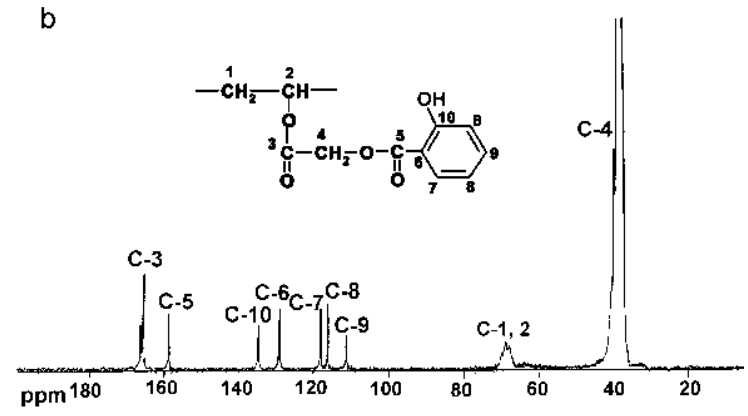

Figure 3. Spectra ${ }^{13} \mathrm{C}-\mathrm{NMR}$ of: a - chloroacet ylated PVA $(98.9 \mathrm{~mol} \%$ of chloroacet ate groups), b - conjugate of PVA-salicylic acid ( $98.9 \mathrm{~mol} \%$ of salicylate groups)

Figure 4 shows the release behaviour of sodium salicy late at $37{ }^{\circ} \mathrm{C}$ and $\mathrm{pH} 8.5$ from three PVA-salicylic acid conjugates with various compositions, containing from 37.8 to $98.9 \mathrm{~mol} \%$ of salicylate groups. From the kinetic curves it follows that the release of the active compound is the quickest in the case of the conjugate with the lowest content of salicylate groups. This seems to be connected with the interaction between the polymer and water. The decreased content of salicylate groups makes the polymer more hydrophilic and consequently facilitates the penetration of hydroxyl ions to ester groups in the conjugate, effectively increasing the relative hydrolysis rates. This is consistent with the results obtained by Babazadeh[13] for conjugates of copolymers 2-hydro xyethyl methacrylate.

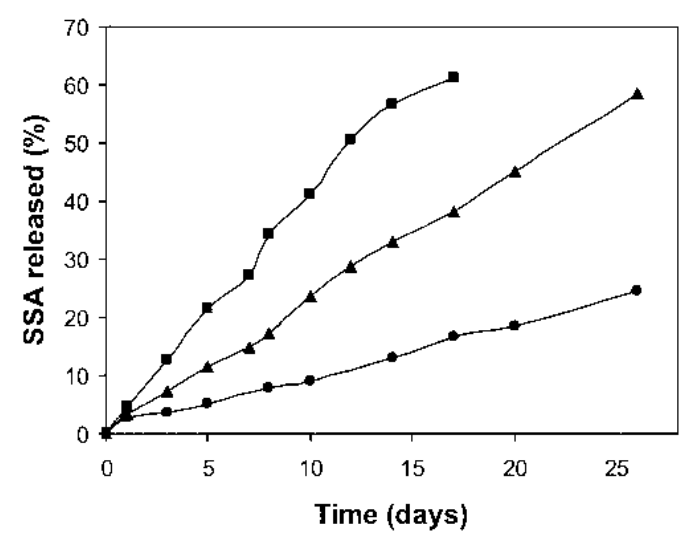

Figure 4. The release of the sodium salicylate with conjugate of PVA-salicylic acid depending on conjugate compositions: (a) $37.8 \mathrm{~mol} \%$ salicylate groups; $(\boldsymbol{\Delta}) 73.6 \mathrm{~mol} \%$ salicylate groups; $(\bullet) 98.9 \mathrm{~mol} \%$ of salicylate groups $\left(\mathrm{pH} 8.5\right.$ at $\left.37^{\circ} \mathrm{C}\right)$

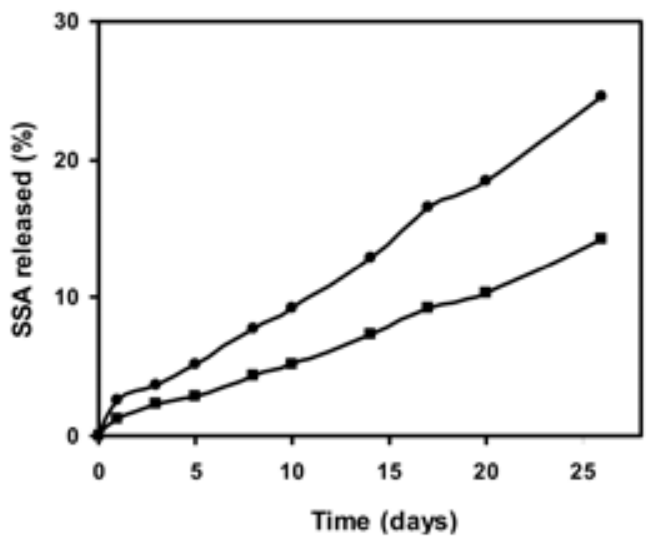

Figure 5. The release of the sodium salicylate with conjugate of PVA-salicylic acid depending on $\mathrm{pH}$ value of reaction environment: (- $) \mathrm{pH}$ 7.6, $(\bullet) \mathrm{pH} 8.5\left(98.9 \mathrm{~mol} \%\right.$ salicylate groups, at $\left.37^{\circ} \mathrm{C}\right)$

Figure 5 shows a typical course of the heterogeneous hydrolysis of PVA-salicylic acid conjugate (containing 98.9 $\mathrm{mol} \%$ of salicylate groups) in slightly alkaline medium from $\mathrm{pH} 7.6$ to 8.5 at $37^{\circ} \mathrm{C}$. The presented results clearly indicate the increase in the release of sodium salicylate with the increase in the alkalinity of reaction medium. The hydrolysis rate of conjugate is the lowest at $\mathrm{pH}$ 7.6. This is consistent with the results obtained by Sanchez-Chaves et al.[14] for the 2-aceto xybenzoic-de xt ran conjugates.

\section{Conclusions}

The studied performed have shown that as a result of the 
reaction between PVA functionalized with chloroacetate groups and sodium salts of salicylic acid a new conjugate PVA-salicylic acid is obtained. The chemical structures of all the modification products of PVA were assessed by means of FT-IR, ${ }^{1} \mathrm{H}-\mathrm{NMR}$ and ${ }^{13} \mathrm{C}-\mathrm{NMR}$ spectroscopy. The hydrolysis of the conjugates was examined under physiological conditions. The results obtained have shown that the procentage of the drug resleased increases with increasing conjugate hydrophilicity and $\mathrm{pH}$ of buffer solutions. The results suggest that the PVA-salicylic acid conjugate can be a useful carrier for controlled release of the drug.

\section{REFERENCES}

[1] Khandare, J.; Minko, T.; Polymer-drug conjugates: Progress in polymeric prodrugs. Prog. Polym. Sci. 2006, 31, 359-397

[2] Hoste, K.; De Winne, K.; Schacht, E. Poly meric prodrugs. Int. J. Pharm. 2004, 277, 119-131

[3] Kim, I.S.; Kim, S.H. Development polymeric nanoparticulate drug delivery system: In vitro characterization of nanoparticles based on super-containing conjugates. Int. J. Pharm. 2002, 245, 67-73

[4] Chang, J.; Du, J.; Zheng, Y. Synthesis and characterization of novel PGA and PLA prodrugs with sulfadiazine and 5-fluorouracil terminal groups. J. Macromol. Sci. 2007, 44, 887-892

[5] D’Souza, A.J.M.; Schowen, R.L.; Topp, F.M. Poly(vinyl pyrrolidone)-drug conjugate: synthesis and release mechanism. J. Control. Release 2004, 94, 91-100

[6] Hang, R.; Kratz, F. Polymer terapeutics: Concepts and applications. Angew. Chem. Int. Ed. 2006, 45, 1198-1215.

[7] Babazadeh, M.; Synthesis and study of controlled release of ibuprofen from the new acrylic type polymers. Int. J. Pharm. 2006, 316, 68-73

[8] Liu, Z.; Rimmer, S. Synthesis and release of 5-fluorouracil from poly(N-vinylpyrrolidone) bearing 5-fluorouracil derivatives. J Control. Release 2002, 81, 91-99

[9] Dvorak, M.; Kopečkova, P.; Kopeček, J. High-molecular weight HPMA copolymer-adriamy cin conjugates. J. Control. Release 1999, 60, 321-322

[10] Bonina, F.P.; Motenegro, L.; Capraiis, P.D.; Palagiano, F.; Trapani, G.; Liso, G. In vitro and in vivo evaluation of poly oxy ethylene indomethacin ester as dermal prodrugs. $J$. Control. Release 1995, 34, 223-232

[11] San Roman, J.; Levenfeld, B. Polymers withpharmacological activity. Macromolecules 1990, 23, 423-427

[12] Bonina, F.P.; Puglia, C.; Barbuzzi, T.; Capraiis, P.D.; Palagiano, F.; Rimoli, M.G.; Saija, A. In vitro and in vivo evaluation of polyoxyethylene ester as dermal prodrugs of ketoprofen, naproxen and diclofenac. Eur. J. Pharm. Sci. 2001, 14, 123-134

[13] Babazadeh, M. Synthesis, characterization and in vitro drug-release properties of 2-hydroxyethyl methacrylate copolymers, J. Appl. Polym. Sci. 2007, 104, 2403-2409

[14] Sanchez-Chaves, M.; Arranz, F.; Diaz, C. Controlled release behaviour of 2-acetoxybenoic acid-dextran conjugates. Macromol. Chem. Rapid. Commun. 1989, 10, 431-433

[15] Duarte, A.R.; Costa, M.S.; Simplicio, A.L.; Cardoso, M.; Duarte, C.M.M. Preparation of controlled release microspheres using supercritical fluid technology for delivery of anti-inflammatory drugs. Int J. Pharm. 2006, 308, 168-174

[16] Won, Ch.Y.; ChCh, Chu.; T, Yu. Synthesis of starch-based drug carrier for the control/release of estrone hormone. $J$. Carbohydrate Research 1997, 32, 239-244

[17] Sanchez-Chaves, M.; Arranz, F.; Diaz, C. Synthesis and characterization of 2-acetoxybenzoic acid-dextran ester conjugates. Macromol. Chem. 1989, 190, 2391-2396

[18] Davaran, S.; Hanaee, J.; Khosravi, A.; Release of 5-amino salicylic acid from acrylic type polymeric prodrugs designed for colon-specific drug delivery. J. Control. Release 1999, 58, 279-287

[19] Jantas; R.; Draczyński, Z.; Stawski, D. Starch functionanalized by chloroacetate groups: coupling of bioactive salicylic acid. Starch, 2007, 59, 366-370

[20] Clarc W.G.; Brater D.C.; Johnson A.R. Goth's Medical Pharmacology, 12 edn. C.V. Mosby Company. St. Louis Washington D.C. 1988

[21] San Roman, J.; Madruga E.L.; Pargoda L. Synthesis and microstructure of polymers from o-methacryloyloxybenzoic acid. J. Polym. Sci. Polym. Chem. Edn. 1987, 25, 203-214

[22] Cai, Q.; Zhu, K.J.; Zhang, J. Salicylic acid and PEG-contained polyanhydrides: synthesis, characterization and in vitro salicy lic acid . J. Drug Delivery. 2005, 12, 97-102

[23] El-Refaie Kenawy, Salem S. Al-Deyab, Mohamed H. El-Newehy, Controlled release of 5-aminosalicylic acid (5-ASA) from new biodegradable polyurethanes.

[24] Molecules 2010, 15, 2257-2268

[25] Davaran, S.; Entezami, A.; Acrylic type polymers containing ibuprofen and indomethacine with difunctional spacer group: synthesis and hydrolysis. J. Control. Release 1997, 47, 41-49

[26] Kim, S.Y.; Shin, I.G.; Lee, Y.M.; Cho, C.S.; Sung, Y.K. Methoxy poly(ethylene glycol) and $\varepsilon$-caprolactone amphiphilic block copolymeric micelle containing indomethacen: II Micelle formation and drug release behaviours. J. Control. Release 1998, 51, 13-22

[27] Davaran, S.; Entezami, A. Hydrophilic copolymers prepared from acrylic type derivatives of ibuprofen containing hydrolyzable thioester bond. Eur. Polym. J. 1998, 34, 187192

[28] Chang, C.H.; Sheu, Y.M.; Hu, W.P.; Wang, L.F.; Chen, J.S. Synthesis and properties of copolymers 2-hydroxyethyl methacrylate-linked nonsteroidal ant-inflammatory agents with methacry lic acid. J. Polym. Sci. Polym. Chem. 1998, 36, $1481-1490$

[29] Zovko, M.; Zorc, B.; Lovrek, M.; Boneschenans, B. Macromolecular prodrugs IX Synthesis of polymerfenoprofen conjugates. Int. J. Pharm. 2001, 228, 129-138

[30] Kim, S.Y.; Chung, C.W.; Hwang, S.J.; Rhee, Y.H. Drug 
release from and hydrolytic degradation of a poly(ethylene glycol) grafted poly(3-hydroxyoctanoate). Int. J. Biol. Macromol. 2005, 36, 84-89

[31] Merwe, T.V.D.; Boneschenans, B.; Zorc, B.; Breytenbach, J.; Zovko, M. Macromolecular prodrugs. X Kinetics of fenoprofen release from PHEA-fenoprofen conjugate. Int. $J$. Pharm. 2002, 241, 223-239

[32] Namazi, B.; Babazadeh, M.; Sarabi, A.; Entezami, A.Synthesis and hydrolysis of acrylic type polymers containing nonstroidal antyinflammantory drugs. J. Polym. Mater. 2001, 18, 301-312
[33] Omata, K.; Nozawa, Y.; Higashide, F. Drug released from cellulose acetic phthalate gel, J. Appl. Polym. Sci. 1977, 21, 2009-2012

[34] Harris, F.W. In Medical Application of Controlled Release. Langer, RS.; Wise, D.L.; Eds.; CRC Boca Raton FL. 1984, p 103

[35] Levenfeld, B.; San Roman, J.; Madrug, E. Polymers with farmacological activity: Synthesis and free radical polymerization of an acrylic derivative of phenacetin. Polymer 31(1990) 160-164 